\title{
LASERTERAPIA EM AFECÇÕES LOCOMOTORAS: REVISÃO SISTEMÁTICA DE ESTUDOS EXPERIMENTAIS
}

\author{
LASER THERAPY ON LOCOMOTOR DISORDERS: A SYSTEMATIC REVIEW \\ OF EXPERIMENTAL STUDIES
}

Artigo de Revisão Sistemática SYSTEMATIC REVIEW ARTICLE Artículo de ReVIsión Sistemática

\section{LASERTERAPIA EN LAS ENFERMEDADES DEL APARATO LOCOMOTOR: REVISIÓN SISTEMÁTICA DE ESTUDIOS EXPERIMENTALES}

Maria Verônica de Souza ${ }^{1}$ (Médica Veterinária)

Micheline Ozana da Silva ${ }^{\top}$ (Fisioterapeuta)

1. Universidade Federal de Viçosa (UFV) Viçosa, MG, Brasil.

\section{Correspondência:}

Departamento de Veterinária, Universidade Federal de Viçosa. Av. PH Rolfs, s/n, Viçosa, Minas Gerais, Brasil. 36570-900

msouza@ufv.br, msouzavet@gmail.com

\section{RESUMO}

Introdução: Ao longo dos anos a fisioterapia tem contribuído para o tratamento das lesões musculotendíneas e osteoarticulares pela utilização de diversos recursos eletrotermofototerapêuticos como, por exemplo, o laser de baixa potência. Objetivo: A finalidade desse estudo foi identificar mediante revisão sistemática, o efeito da laserterapia de baixa potência em afecções do sistema locomotor de ratos, com destaque para a dose de energia e o comprimento de onda utilizado, assim como para a resposta ao tratamento. Métodos: Foram consultadas as bases de dados Medline, Lilacs, PEDro e SciELO, entre janeiro de 2005 a maio de 2013, utilizando os termos "tendinopathy", "laser therapy", "rats", "tendon", "muscle", "bone", "low-level laser therapy", assim como "tendinopatia", "laserterapia", "ratos", "tendão", "músculo", "osso" e "laser de baixa potência". Apenas foram incluídos estudos experimentais publicados nos idiomas português e inglês, que induziram lesão em tendões, músculos, ossos e/ou articulação, tratada com laserterapia, associada ou não a outros tratamentos. Resultados: Foi encontrado um total de 30 estudos experimentais nas bases de dados consultadas, dos quais 15 (50\%) foram realizados em tendão, 10 (33,33\%) em músculo e cinco (16,66\%) em osso e/ou articulação. As doses de energia mais comumente utilizadas foram de $3 \mathrm{~J}(26,66 \%)$ e $1 \mathrm{~J}(16,66 \%)$. Já os comprimentos de onda foram de 904 nm $(21,21 \%)$ e 830 nm (21,21\%). Conclusão: A informação mais expressiva obtida com a terapia foi a diminuição da resposta inflamatória (36,66\%) em lesões ortopédicas agudas.

Descritores: lasers; tendinopatia; miopatia; artropatia; defeito ósseo

\section{ABSTRACT}

Introduction: Physiotherapy has long been contributing for the treatment of musculotendinous and osteoarticular lesions by using several electrothermal and phototherapy resources, such as low-level laser. Objective: The aim of this study was to identify, through a systematic review, the effect of low-level laser therapy on locomotor system diseases in rats, mainly the energy dose and wavelength applied, as well as the response to the treatment. Methods: Databases Medline, Lilacs, PEDro and SciELO were consulted from January 2005 to May 2013, using the terms "tendinopathy", "laser therapy", "rats", "tendon", "muscle," "bone", "low-level laser therapy", as well as "tendinopatia", "laserterapia", "ratos", "tendão", " músculo", "osso" and "laser de baixa potência". Only experimental studies published in Portuguese and English and that induced lesion in tendons, muscles, bones and/or joints, treated with laser therapy, were included, combined or not with other treatments. Results: A total of 30 experimental studies were found in the databases consulted, out of which 15 (50\%) were carried out on tendon, 10 (33.33\%) on muscle, and five (16.66\%) on bone and/or joint. The energy doses most frequently used were $3 \mathrm{~J}$ (26.66\%) and $1 \mathrm{~J}$ (16.66\%), while the most frequent wavelengths were $904 \mathrm{~nm}$ (21.21\%) and $830 \mathrm{~nm}$ (21.21\%). Conclusion: The most expressive information obtained with this therapy was the decrease in inflammatory response (36.66\%) in acute orthopaedic lesions.

Keywords: lasers; tendinopathy; myopathy; arthropathy; bone defect

\section{RESUMEN}

Introducción: A lo largo de los años la fisioterapia ha contribuido para el tratamiento de lesiones músculo-tendinosas y osteoarticulares, por la utilización de diferentes recursos electrotérmicos y fototerapéuticos como, por ejemplo, el láser de baja potencia. Objetivo: El propósito de ese estudio fue identificar por medio de revisión sistemática, el efecto de la laserterapia de baja potencia en los trastornos del aparato locomotor en ratones, destacando la dosis de energía y la longitud de onda utilizada, así como la respuesta al tratamiento. Métodos: Se ha consultado las bases de datos Medline, Lilacs, PEDro y SciELO, entre enero de 2005 y mayo de 2013, utilizando los términos "tendinopathy", "laser therapy", "rats", "tendon", "muscle", "bone," "low-level laser therapy", así como "tendinopatia", "laserterapia", "ratos", "tendão", "músculo", "osso" y "laser de baixa potência". Sólo fueron incluidos estudios de investigación publicados en portugués y en inglés, que indujeron lesiones en los tendones, músculos, huesos y/o articulaciones, tratados con la laserterapia, con o sin la asociación con otros tratamientos. Resultados: Hubo un total de 30 estudios experimentales en las bases de datos evaluadas, de los cuales 15 (50\%) se realizaron en el tendón, 10 (33,33\%) en el músculo y cinco (16,66\%) en el hueso y/o articulación. Las dosis de energía más utilizadas 
fueron $3 J(26,66 \%)$ y $1 J(16,66 \%)$. Por otra parte, las longitudes de onda fueron $904 \mathrm{~nm}(21,21 \%)$ y $830 \mathrm{~nm}(21,21 \%)$. Conclusión: La información más importante obtenida con la terapia fue la reducción de la respuesta inflamatoria (36,66\%) en lesiones ortopédicas agudas.

Descriptores: láseres; tendinopatía; miopatía; artropatía; defecto óseo

\section{INTRODUÇÃO}

Existe uma busca incessante por modalidades terapêuticas que promovam uma recuperação mais rápida de lesões musculoesqueléticas e osteo-articulares tanto na espécie humana, quanto animal. Os tratamentos convencionais objetivam inicialmente o alívio da dor e a resolução do processo inflamatório, porém os resultados nem sempre são satisfatórios. As opções de tratamento comumente utilizadas são a administração de corticosteroide ou drogas anti-inflamatórias não esteroidais (Aines), modalidades de calor como a fonoforese, iontoforese e ultrassom terapêutico, massagem de fricção transversal, ondas de choque, atividade física controlada, laserterapia, entre outras ${ }^{1-4}$. Os esteroides e Aines são benéficos apenas na fase aguda das lesões ${ }^{5}$, entretanto, para que uma terapia seja considerada eficiente, deve reduzir não apenas a inflamação aguda, mas também proporcionar reparação tecidual em curto período de tempo ${ }^{6}$, e promover uma recuperação do tendão o mais próxima da sua funcionalidade fisiológica.

O laser, amplificação da luz mediante emissão estimulada de radiação, é uma terapia que vem sendo empregada mundialmente para o tratamento de diversas afecções. Desde 1960, pesquisas são realizadas para investigar a ação terapêutica do laser de baixa potência (LBP) ou intensidade2, também denominado de "laser frio", "soft laser", "fotoestimulação ou bioestimulação"7. Os aparelhos existentes podem ser classificados segundo o tipo do seu meio ativo (um amplificador óptico), que pode ser sólido, líquido e semicondutor de gás ${ }^{8}$. O LBP pode emitir uma radiação visível ou invisível (infravermelho), com comprimentos de onda que variam entre $600 \mathrm{~nm}$ a $1.000 \mathrm{~nm}^{7,9}$, sendo atérmico e não invasivo. 0 tipo de radiação está relacionado com a via de ação pretendida com a aplicação do laser ${ }^{10}$. Para as afecções musculoesqueléticas são disponibilizados os lasers à base de arseneto de gálio (Ga-As) e hélio-neônio (He-Ne) ${ }^{6,11}$. Também existem estudos utilizando o laser à base de arseneto de gálio associado a alumínio (GaAlAs) ${ }^{12-14}$.

Entre os benefícios resultantes da laserterapia podem ser citados o efeito analgésico em lesões ósseas, musculares e tendíneas agudas ou crônicas ${ }^{15}$, vasodilatação e proliferação de microvasos, com possível aumento da quantidade de oxigênio no tecido ${ }^{16}$, proliferação epitelial, endotelial e fibroblástica, aumento da síntese de colágeno (COL) e da atividade fagocitária, o que resultará na aceleração do processo de reparação ${ }^{17}$, além da liberação de citocinas que irão reduzir a reação inflamatória ${ }^{18}$. A resposta à bioestimulação depende diretamente do comprimento da onda, da densidade de energia [Joules por $\mathrm{cm}^{2}$ $\left(\mathrm{J} / \mathrm{cm}^{2}\right)$ ], e da potência, além do tempo da aplicação ${ }^{15,19,20}$. A penetração da radiação depende do comprimento da onda, da forma que, quanto menor o comprimento da onda maior será a sua ação ${ }^{15}$. Existe uma dose ideal de aplicação da luz, de modo que doses mais baixas ou mais altas do que a ideal, podem ocasionar um resultado indesejado ${ }^{7}$.

Baseado em pesquisas, a Associação Mundial para Terapia com Laser padronizou algumas dosagens para humanos, de forma que, para cada tipo de tecido a ser tratado, há doses e comprimentos de ondas indicados ${ }^{21}$. As propriedades do laser permitem que a luz penetre na superfície da pele, e a energia gerada transferida diretamente à célula-alvo, sem causar dano ao tecido ${ }^{15}$. Os mecanismos de ação do laser, em nível molecular e celular, ainda não estão bem esclarecidos ${ }^{14}$. A radiação visível atua pela via mitocondrial, já a invisível tem uma ação sobre cromóforos da membrana celular ${ }^{22}$. Um provável mecanismo de ação da terapia no processo inflamatório é a modulação na síntese de citocinas, como as interleucinas (IL) 1 $\beta, 6$ e 10 1,23.

Modelos animais são utilizados experimentalmente para obtenção de resultados que demonstrem a eficácia da terapia, e que possam ser extrapolados para os seres humanos ${ }^{23-26}$. Para isso devem permitir a reprodução de lesões semelhantes às observadas no homem, que possam ser observadas, diagnosticadas e tratadas ${ }^{27}$. Os ratos são considerados excelentes modelos experimentais para a espécie humana, devido a sua semelhança anatômica e fisiológica. Como exemplo são destacados os tendões ${ }^{28}$. Essa similaridade possibilita que as informações obtidas em pesquisas envolvendo diferentes tecidos e órgãos, possam ser utilizadas para o tratamento de afecções que afetam o homem. Adicionalmente, a resistência desses animais em comparação com outras espécies ${ }^{29}$ é um aspecto positivo a ser considerado em estudos dessa natureza.

Essa revisão sistemática parte do princípio que a laserterapia apresenta efeito positivo sobre a reação inflamatória que ocorre na fase inicial de afecções locomotoras, ainda que exista variabilidade nas doses e no comprimento de onda utilizados em estudos controlados. Nesse contexto, o objetivo do presente estudo foi identificar, por meio dos artigos científicos, o efeito da laserterapia de baixa potência, em problemas ortopédicos de ratos, considerado modelo experimental para seres humanos, com destaque para a dose de energia e para o comprimento de onda utilizado, assim como para a resposta ao tratamento.

\section{MÉTODOS}

Foi realizada uma busca sistemática no período compreendido entre janeiro de 2005 a maio de 2013, para realização de uma revisão sobre experimentos que utilizaram laserterapia no tratamento de afecções locomotoras em ratos. Para isso, foram consultadas as bases de dados Medline, Lilacs, PEDro e SciELO. Os termos utilizados para a busca dos estudos foram "tendinopathy", "laser therapy", "rats", "tendon", "muscle", "bone", "low-level laser therapy", assim como "tendinopatia", "laserterapia", "ratos", "tendão", "músculo", "osso" e "laser de baixa potência". Como critério de inclusão foi considerado estudos experimentais, publicados na língua portuguesa e inglesa, que realizaram indução de lesões tendínea, muscular, óssea e/ou articular, que foram tratadas com LBP, associadas ou não a outros tratamentos. Foram excluídos estudos que não utilizaram a laserterapia; experimentos realizados com lesões odontológica, oftalmológica, dermatológica e musculatura lisa; artigos de revisão com seres humanos ou outras espécies animais, e aqueles publicados fora do período preconizado na presente revisão. Nos artigos selecionados foram avaliados os seguintes itens: local da lesão, dose de energia, comprimento de onda empregado e principais resultados obtidos. Os resultados foram analisados por estatística descritiva.

Para a inclusão dos artigos científicos seguiu-se um fluxograma de etapas (Figura 1), conforme recomendação do "PRISMA Flow chat"30. Resumidamente, foi realizada a identificação de registros de utilização da laserterapia nas bases de dados selecionadas, mas como outros artigos foram encontrados além das bases preconizadas, esses foram 


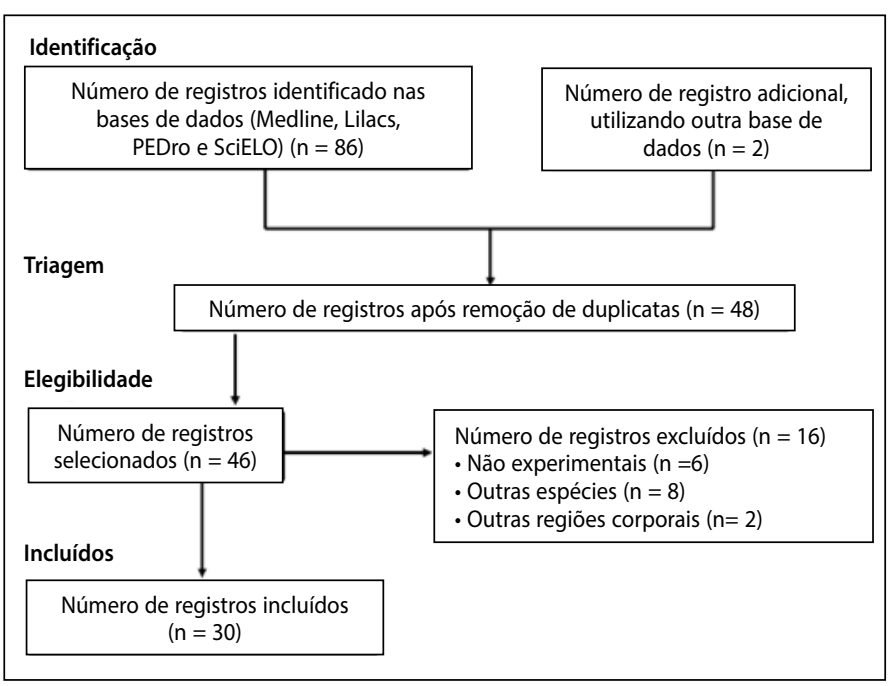

Figura 1. Fluxo de inclusão de registro dos artigos durante diferentes etapas da revisão sistemática.

inicialmente mantidos no processo seletivo. Essa etapa foi realizada por dois pesquisadores, que se basearam no título e resumo para a inclusão dos artigos. Na sequência foi realizada a triagem, onde foram eliminados os registros em duplicata. Na etapa seguinte foi considerada a elegibilidade dos artigos, sendo considerada a metodologia empregada, a utilização de estudo randomizado e controlado, e eliminados aqueles que não correspondiam a estudos experimentais, assim como a outras espécies e não relacionados com afecções locomotoras em ratos. Finalmente foram apenas incluídos os registros realmente presentes nas bases de dados eleitas, consideradas como referências em algumas revisões sistemáticas, e depois de todo o conteúdo do artigo ser consultado.

Essa revisão está vinculada ao projeto de pesquisa aprovado pela Comissão de Ética no Uso Animal (CEUA) da Universidade Federal de Viçosa (Processo 108/2011).

\section{RESULTADOS}

Considerando os critérios de inclusão adotados, foram encontrados um total de 30 estudos nas bases de dados consultadas, dos quais quinze (50\%) foram realizados em tendão 3,4,6,9,22-24,31-38, dez (33,33\%) em musculos ${ }^{12,14,39-45}$, e cinco $(16,66 \%)$ em osso e/ou articulação $0^{13,25,46-48}$. A Tabela 1 apresenta detalhes sobre esses estudos.

Foram utilizados diferentes tipos de LBP, com diferentes ajustes. As doses de energia mais comumente utilizadas foram de $3 \mathrm{~J}(26,66 \%)$ e $1 \mathrm{~J}(16,66 \%)$. Já os comprimentos de onda foram $904 \mathrm{~nm}(21,21 \%), 830$ nm (21,21\%), 810 nm (12,12\%), 780 nm (12,12\%) e 660 nm (9,09\%). Três estudos $(9,09 \%)$ associaram diferentes comprimentos de onda (670 e $904 \mathrm{~nm}, 830$ e $904 \mathrm{~nm}$ e 660 e $830 \mathrm{~nm}$ ).

Os resultados mais expressivos obtidos com a terapia foram: diminuição da resposta inflamatória mediante redução de células inflamatórias e modulação de mediadores da inflamação (36,66\%), e o aumento da síntese de colágeno (13,33\%). Alguns dos demais resultados (50,01\%) mencionados foram: angiogênese; redução do estresse oxidativo, do colágeno e da fibrose; aumento do tecido de granulação, da remodelação e síntese óssea, assim como da expressão da proteína da diferenciação miogênica (MyoD) e do fator de crescimento endotelial vascular (VEGF). Também foi relatada a redução do fator de crescimento transformante beta (TGF- $\beta$ ) e da concentração da creatina quinase (CK) e do lactato sanguíneo, com aumento da força muscular; melhora das propriedades biomecânicas do tendão e da função locomotora como um todo.

\section{DISCUSSÃO}

Vários estudos foram realizados com a finalidade de se obter meIhor compreensão dos efeitos da terapia com laser. Segundo Carvalho et al. ${ }^{44}$ não há relatos de efeitos colaterais na laserterapia. Salate et al. ${ }^{31}$ observaram na fase inflamatória e proliferativa da lesão parcial do tendão calcanear comum de ratos (TCC), também conhecido como tendão de Aquiles, que a laserterapia induz a angiogênese in vitro e in vivo. Fillipin et al. ${ }^{6}$ observaram em lesão induzida por trauma no TCC, redução na reposta inflamatória e da fibrose em avaliação histopatológica realizada sete dias da indução da lesão, assim como do estresse oxidativo, pelo teste das substâncias reativas ao ácido tiobarbitúrico, onde foi observado o aumento da atividade da superóxido dismutase aos 14 e 21 dias. Além da diminuição da fase inflamatória, Laraia et al..23 demonstraram redução das citocinas pró-inflamatórias (IL-1 $\beta$ e IL-6) e aumento da anti-inflamatória (IL-10), durante a recuperação do tendão calcanear de ratos submetidos à ruptura parcial. Outros estudos também demonstraram modulação de citocinas pró e anti-inflamatórias, reduzindo a resposta inflamatória ${ }^{4,9,23,33,34,47}$. Portanto, apesar de terem sido incluídos estudos randomizados e controlados, muitos deles buscaram respostas diferentes aos efeitos da laserterapia, tanto na fase inflamatória, proliferativa e de remodelação ou modelação das distintas afecções locomotoras registradas. Para isso foram utilizadas diferentes técnicas de diagnóstico, algumas delas com alta especificidade e sensibilidade. Nesse contexto, houve uma variabilidade na resposta ao tratamento, uma vez que distintos fatores podem influenciar os resultados obtidos. Esse aspecto deve ser considerado ao se utilizar um artigo científico como base para realização de outros experimentos.

A angiogênese citada como resultante da laserterapia é importante para nutrição e oxigenação tecidual. Em tendões bem vascularizados, o tecido pode se recuperar rapidamente, mas naqueles que são hipo ou avasculares, o processo de reparação é mais demorado, aumentando as chances de recidiva, caso o animal seja exposto a uma força prejudicial. A elevação da superóxido dismutase descrita por Fillipin et al. ${ }^{6}$ em TCC após tratamento com LBP também é uma resposta desejável, devido ao efeito anti-oxidante dessa enzima. Sabe-se que o estresse oxidativo é um potente fator patogênico que acarreta alterações degenerativas, incluindo fibrose ${ }^{39}$. Na realidade, a angiogênese é uma resposta fundamental, particularmente na fase inicial de uma lesão em uma afecção locomotora, mas é necessário que a mesma diminua após um incremento inicial, já que a sua persistência interfere no processo de regeneração dos tecidos. Esse é outro aspecto que deve ser considerado ao se utilizar e comparar os resultados obtidos nessas pesquisas.

Guerra et al. ${ }^{34}$ observaram que a radiação do LBP promove aumento da metaloproteinase da matriz (MMP)-2 em animais com o TCC parcialmente rompidos. Marcos et al. ${ }^{33}$ demonstraram haver redução das MMPs-3, 9 e 13 nesse mesmo tendão lesionado por colagenase. Segundo os autores, a expressão das MMPs-1 e 13 está associada à tendinopatias, enquanto que a MMP-3, assim como a MMP-13, quando em altas concentrações, ocasionam a decomposição do COL I, podendo causar danos ao tendão. A MMP-1 é uma das mais importantes colagenases e atua decompondo o colágeno, assim como a MMP-13, enquanto a MMP-3 atua degradando substratos de proteínas da matriz extracelular ${ }^{33,49}$. Para que ocorra remodelação da matriz tendínea é necessária que haja equilíbrio entre as MMPs e seus inibidores (TIMPs), visto que essas enzimas são essenciais à migração de células para a matriz extracelular, além de remover o colágeno e os componentes da matriz que foram desnaturados durante o processo lesivo. Caso esse colágeno não seja removido se formará um tecido desorganizado, com inadequada matriz extracelular. 
Tabela 1. Características dos estudos incluídos no período de janeiro de 2005 a maio de 2013, em lesões induzidas experimentalmente em ratos, tratadas com laserterapia.

\begin{tabular}{|c|c|c|c|c|}
\hline Autor & Estrutura lesionada & $\begin{array}{l}\text { Comprimento } \\
\text { de onda (nm) } \\
\end{array}$ & Dose (J) & Resumo dos resultados obtidos \\
\hline Wood et al. $(2010)^{3}$ & TCC & 830 & 0,12 & $\begin{array}{l}\text { Aumento da síntese de COL I, quando associada ao utrassom } \\
\text { terapêutico. }\end{array}$ \\
\hline Xavier et al. $(2010)^{4}$ & TCC & $880 \pm 10$ & 7,5 & Redução na expressão de IL-1 $\beta$, IL-6, COX-2 e do TNF. \\
\hline Fillipin et al. $(2005)^{6}$ & TCC & 904 & 5 & $\begin{array}{l}\text { Redução do processo inflamatório, da concentração de colágeno } \\
\text { e da fibrose. Aumento da atividade da superóxido dismutase. }\end{array}$ \\
\hline Pires et al. $(2011)^{9}$ & TCC & 780 & 7,7 & Redução na expressão de mediadores IL-6, COX-2 e TGF- $\beta$. \\
\hline Lopes-Martins et al. $(2006)^{12}$ & Músculo tibial anterior & 655 & 0,$5 ; 1$ e 2,5 & Redução dos níveis de CK e aumento da força muscular. \\
\hline Pretel et al. $(2007)^{13}$ & Defeito ósseo na mandíbula & 780 & 1,4 & Aceleração da remodelação e da síntese óssea. \\
\hline Assis et al. $(2013)^{14}$ & Músculo tibial anterior & 808 & 1,4 & $\begin{array}{l}\text { Aumento da expressão da MyoD e do VEGF. Redução } \\
\text { do TGF- } \beta \text { e da fibrose. }\end{array}$ \\
\hline Arruda et al. $(2007)^{22}$ & TCC & 670 e 904 & 3 & Organização das fibras de colágeno. \\
\hline Laraia et al. $(2012)^{23}$ & TCC & 660 & 6 & Redução das IL-1 $\beta$ e IL-6, e aumento da IL-10. \\
\hline Nouruzian et al. $(2012)^{24}$ & TCC & 632.8 & 2,$9 ; 11,5$ e 4,3 & $\begin{array}{l}\text { Melhora nas propriedades biomecânicas do tendão } \\
\text { (carga e tensão máximas). }\end{array}$ \\
\hline Pallotta et al. $(2012)^{25}$ & Artrite da articulação fêmoropatelar & 810 & $1,3,6$ e 10 & $\begin{array}{c}\text { Redução de polimorfonucleares, IL-1 e 6, e da PG E } E_{2 ;} \\
\text { aumento da COX-1 e 2. }\end{array}$ \\
\hline Ramos et al. $(2012)^{26}$ & Músculo tibial anterior & 810 & $1,3,6$ e 9 & Melhora da função locomotora, mediante avaliação de apoio. \\
\hline Salate et al. $(2005)^{31}$ & TCC & 660 & 2,5 e 10 & Angiogênese. \\
\hline Oliveira et al. $(2009)^{32}$ & TCC & 830 & 4 & Organização das fibras de colágeno. \\
\hline Marcos et al. $(2012)^{33}$ & TCC & 810 & 1 e 3 & $\begin{array}{l}\text { Diminuição da resposta inflamatória, por redução } \\
\text { das MMPs-3, } 9 \text { e } 13 .\end{array}$ \\
\hline Casalechi et al. $(2012)^{34}$ & TCC & 780 & 7,5 & $\begin{array}{l}\text { Redução da resposta inflamatória pela modulação da IL-10, } \\
\text { VEGF e MMP-1 e 13. }\end{array}$ \\
\hline Guerra et al. $(2013)^{35}$ & TCC & 830 & 4 & Aumento da síntese de COL I e III e ativação da MMPs-2. \\
\hline Joensen et al. $(2012)^{36}$ & TCC & 904 & 3 & Sem resposta ao tratamento \\
\hline Lima et al. $(2012)^{37}$ & TCC & 658 & 3 e 6 & Redução na deposição de fibras colágenas. \\
\hline Barbosa et al. $(2013)^{38}$ & TCC & 660 e 830 & 7,0 & $\begin{array}{c}\text { Aumento na deposição de COL I, quando associada } \\
\text { a plasma rico em plaquetas. }\end{array}$ \\
\hline Rizzi et al. $(2006)^{39}$ & Músculo gastrocnêmio & 904 & 5 & $\begin{array}{l}\text { Redução da resposta inflamatória e do colágeno; } \\
\text { bloqueio da ativação do NF-kB, e da expressão de iNOS. }\end{array}$ \\
\hline Leal Junior et al. $(2010)^{40}$ & Músculo tibial anterior & 904 & 0,$1 ; 0,3 ; 1$ e 3 & Redução dos níveis de CK e lactato no sangue. \\
\hline Falcai et al. $(2010)^{41}$ & Músculo gastrocnêmio & 960 & 2 & Angiogênese e melhor organização tecidual. \\
\hline Moreira et al. $(2011)^{42}$ & Músculo gastrocnêmio & 830 e 904 & 5 & $\begin{array}{l}\text { Aumento da síntese de COL, sete dias após lesão, } \\
\text { e redução aos } 14 \text { dias. }\end{array}$ \\
\hline Silveira et al. $(2012)^{43}$ & Músculo gastrocnêmio & 904 & 5 & $\begin{array}{l}\text { Redução do estresse oxidativo, mediante redução da superóxido } \\
\text { dismutase, ânion superóxido e dos níveis de ácido tiobarbitúrico. }\end{array}$ \\
\hline Carvalho et al. $(2013)^{44}$ & Músculo tibial anterior & 810 & 3 & Redução na expressão da COX-1 e 2 e da PGE 2. \\
\hline Fangel et al. $(2011)^{46}$ & $\begin{array}{l}\text { Indução de osteopenia e falha } \\
\text { óssea na tíbia }\end{array}$ & 830 & 1,7 & Aumento do tecido de granulação e neoformação óssea. \\
\hline Alves et al. $(2011)^{47}$ & $\begin{array}{l}\text { Artrite da articulação } \\
\text { femorotibiopatelar }\end{array}$ & 780 & 7,7 & $\begin{array}{l}\text { Redução das células inflamatórias mononucleares, e aumento } \\
\text { dos condroblastos e osteoblastos. }\end{array}$ \\
\hline Rodrigues et al. $(2013)^{48}$ & Músculo tibial anterior & 660 & 10 e 50 & $\begin{array}{l}\text { Redução do infiltrado inflamatório e aumento da expressão } \\
\text { da MyoD e VEGF. }\end{array}$ \\
\hline Fernandes et al. $(2013)^{52}$ & Terço proximal da tíbia & 830 & 2,8 & $\begin{array}{l}\text { Redução da resposta inflamatória; angiogênese; } \\
\text { melhor organização tecidual e osteogênese. }\end{array}$ \\
\hline
\end{tabular}

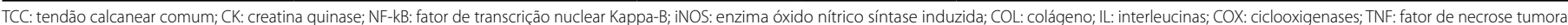
TGF- $\beta$ : fator de crescimento transformante beta; VEGF: fator de crescimento endotelial vascular; MMP: metaloproteinase da matriz; PG: prostaglandina; MyoD: diferenciação miogênica.

Ainda com relação às MMPs, embora o incremento das MMP-2 e MMP-9 seja importante para a degradação do colágeno desnatura, a elevação excessiva pode agravar a lesão, já que ambas estimulam a produção do TNF- a e IL-1ß. Portanto, a redução da gelatinase MMP-9 no TCC lesionado por colagenase, observada por Marcos et al. ${ }^{33}$, revela um efeito positivo da laserterapia.

Ainda no que se refere ao efeito terapêutico do laser em estrutura tendínea, maior organização das fibras colágenas ao longo do eixo longitudinal do TCC é observada após lesão parcial ${ }^{32}$. A terapia com laser também promove aumento na resistência do tendão ${ }^{24}$. Por outro lado, após lesão do TCC com utilização de guilhotina, Joensen et al. ${ }^{36}$ não observaram resposta à laserterapia, mas identificaram aumento do edema na avaliação ultrassonográfica realizada 23 horas após o tratamento. Esses resultados demonstram a importância em se levar em consideração a forma de indução da lesão em estudos controlados e randomizados. Existem vários modelos de indução de lesão nas estruturas do aparelho locomotor, e pode-se encontrar diferentes respostas à laserterapia.

Uma melhor organização das fibras colágenas é fundamental para a resistência do tendão à tração, o que permite transmissão da força gerada pela contração muscular. Entretanto, apesar de ter observado resposta satisfatória após aplicação do laser como, por exemplo, redução do estresse oxidativo, Fillipin et al. ${ }^{6}$ verificaram que a laserterapia reduz a concentração de colágeno 21 dias após lesão traumática no TCC. Os autores associam esse achado à provável redução do processo inflamatório. É fundamental levar em consideração nesses estudos científicos o tempo de avaliação da resposta à laserterapia. O processo de reparação de diversos tecidos em ratos é bastante 
rápido, e dependendo do período de estudo podem-se encontrar diferentes respostas ao tratamento. Esse aspecto também é essencial ao se selecionar um experimento previamente realizado para se tomar como base para futuras pesquisas.

Quando utilizado em lesão muscular, ficou demonstrado que a terapia promove redução da fadiga muscular pela diminuição da concentração do lactato sanguíneo, conforme mencionado por Leal Junior et al. ${ }^{40}$. Esse efeito pode está relacionado com a ação vasodilatadora do LBP, que permite aumentar a disponibilidade de oxigênio no tecido ${ }^{16}$ e, consequentemente, a conversão do excesso de lactato em piruvato, que por sua vez é metabolizado no ciclo de Krebs.

Rizzi et al. ${ }^{39}$ avaliaram o efeito do laser no músculo gastrocnêmio submetido a trauma pelo método guilhotina. Os autores encontraram redução da expressão de iNOS, e bloqueio da ativação do NF-kB. A ¡NOS é produzida a partir de estímulos provenientes de várias células do organismo, como por exemplo, macrófagos e neutrófilos. Essa isoenzima libera óxido nítrico, que quando em altas concentrações pode gerar processos patológicos. Nesse contexto, sua redução pela laserterapia após lesão traumática é um achado satisfatório. O NF-kB é um fator de transcrição nuclear que regula a expressão de genes que estão relacionados com a inflamação.

A IL-1 $\beta$, o TNF-a e os produtos de degradação de matriz extracelular desencadeiam sinais a esse fator, que normalmente encontra-se em estado inativo no citoplasma da célula. Entretanto, de acordo com Marcu et al. ${ }^{50}$, uma vez ativado, controla a expressão de várias citocinas reguladoras da inflamação como, por exemplo, as IL-6, 8 e 10, o fator estimulante de colônias de granulócitos e macrófagos e a proteína quimiotativa para monócitos que, por sua vez, desencadeiam a degradação enzimática da matriz pelas MMPs-1, 3 e 13, e agrecanases. Portanto, o estudo desses autores sugere que a redução da sinalização ao NF-kB revela eficácia da laserterapia.

Redução da resposta inflamatória e dos níveis de COX-2, com aumento da expressão da MyoD foram obtidos após laserterapia em lesão realizada no músculo tibial anterior ${ }^{51}$. Embora Moreira et al. ${ }^{42}$ tenham observado uma tendência a maior deposição de COL I no músculo gastrocnêmio lesionado cirurgicamente mediante incisão longitudinal, após 14 dias de tratamento com LBP não houve diferença entre grupos tratado com Ga-As $(p=0,15)$ e GaAlAs $(p=0,19)$, em relação ao grupo controle (não submetido a radiação).

Com relação à maior deposição de colágeno tipo I no músculo gastrocnêmio tratado com laserterapia ${ }^{33}$, sabe-se que este deve ser o predominante no músculo de ratos, o que é fundamental devido à sua elasticidade, mas também pela sua ação na estabilização da arquitetura tecidual. Esse colágeno é denominado intersticial, uma vez que forma estrutura fibrilares no espaço intercelular ${ }^{52}$. Entretanto, conforme mencionado anteriormente, não houve diferença significativa quando comparado grupos tratado e controle. Na realidade, segundo Fillipin et al. ${ }^{6}$ existe controvérsia sobre o efeito do laser sobre o metabolismo do colágeno. Rizzi et al. ${ }^{38}$ observaram, inclusive, diminuição do colágeno sete a 14 dias após a sua elevação ocasionada por trauma no músculo gastrocnêmio. 0 mesmo foi relatado 21 dias após lesão traumática em TCC ${ }^{6}$. Estudos recentemente publicados em outra espécie (equina) e em outro tecido (pele) demonstraram que, dependendo da fase do processo de regeneração ou cicatrização, a expressão de colágenos determinada mediante as técnicas como histomorfometria ${ }^{53}, \mathrm{RT}$-qPCR ${ }^{54}$ e imunoistoquímica ${ }^{55}$, pode não se mostrar diferença entre grupos tratados e não tratados, inclusive utilizando uma terapia regenerativa como o plasma rico em plaquetas. O mesmo pode acontecer com a laserterapia e em ratos. Acredita-se que, embora o tecido em estudo esteja aparentemente cicatrizado, alterações químicas e mecânicas ainda irão ocorrer de forma que um maior tempo de avaliação seria necessário para realmente demonstrar ou não a eficácia de uma terapia.

As ciclo-oxigenases são enzimas responsáveis por clivar o ácido araquidônico. A COX-1 é expressa em vários tecidos e resulta na síntese de prostaglandinas relacionadas com reações fisiológicas em diferentes tecidos. Por outro lado, os produtos resultantes da quebra do ácido araquidônico pela COX-2 desencadearão uma resposta inflamatória a partir das citocinas e mediadores da inflamação. Essa isoforma (COX-2) está envolvida em diversos mecanismos celulares, como angiogênese, proliferação e prevenção de apoptose. Estudo recentemente publicado em artrite da articulação fêmoropatelar ${ }^{25}$ revelou que o laser aumenta a expressão gênica de COX-1 e 2, o que é um efeito indesejável. Por outro lado, Rodrigues et al. ${ }^{45}$ observaram redução da COX-2 em músculo tibial anterior tratado com laserterapia. Não há dúvida que a diminuição dessa ciclo-oxigenase é um achado favorável, já que apesar da resposta inflamatória fazer parte do processo de reparação fisiológico das estruturas musculotendíneas, quando persistente pode estimular a expressão de genes pró-fibróticos. Resultados contraditórios são mencionados conforme o tipo de tecido estudado, dose de energia e o comprimento de onda utilizado. Portanto, pesquisas ainda são necessárias para investigar a produção de mediadores anti-inflamatórios resultantes das ciclo-oxigenases induzidas no tratamento com LBP.

Alves et al. ${ }^{47}$ também observaram redução das células inflamatórias, mas também aumento da quantidade de condroblastos e osteoblastos em animais com artrite reumatóide da articulação femorotibiopatelar. Os condroblastos são células que secretam componentes da matriz extracelular para a formação da estrutura cartilaginosa, enquanto os osteoblastos participam da síntese dos componentes da matriz óssea, expressam fosfatase alcalina, enzima que permite a mineralização da matriz, e inclusive secretam fatores de crescimento. Fangel et al. ${ }^{46} \mathrm{e}$ Fernandes et al. ${ }^{48}$ também descreveram aumento da síntese óssea após tratamento com laserterapia na tíbia de ratos. Os autores observaram, mediante avaliação histopatológica, neoformação óssea particularmente na análise realizada cinco dias após lesão traumática e tratamento. Ocorreu aumento da expressão dos genes para o RNAm da fosfatase alcalina, da osteocalcina, e do Runex (fator de transcrição associado com a diferenciação osteoblástica). A fosfatase alcalina e osteocalcina são marcadores de formação óssea, e sua elevação representa melhora na constituição óssea. Ainda com relação a enzimas, a redução da concentração sanguínea de CK observada em dois estudos adicionados a essa revisão sistemática foi adequada para uma melhor resposta na força muscular. Como a CKé marcadora muscular, sua diminuição indica redução da lesão tecidual ${ }^{12}$.

Fernandes et al. ${ }^{48}$ também relataram como efeito positivo da laserterapia em lesão óssea, a modulação da resposta inflamatória (por recrutamento de células inflamatórias), assim como o aumento do número de fibroblastos e de capilares sanguíneos. Ocorreu melhor organização tecidual, pela presença de tecido de granulação. Resultados semelhantes, no que se refere ao aumento da síntese óssea e modulação da resposta inflamatória, também foram observados após laserterapia em defeito realizado na mandíbula ${ }^{13}$. Esses resultados demonstram que não apenas tecidos superficiais como profundos podem ser beneficiados com o uso da laserterapia. Entretanto, conforme previamente mencionado, não há consenso de qual dose e comprimento de onda seriam mais adequados no tratamento das afecções musculotendíneas e osteo-articulares. Conforme apresentado na Tabela 1, doses acima de 4 J estão associadas à redução do processo inflamatório. Utilizando doses de 1, 3, 6 e 9 J no tratamento de lesão muscular, 
Ramos et al. ${ }^{26}$ demonstraram que ratos tratados com dose de $3 \mathrm{~J}$ apresentaram melhora na funcionalidade do músculo tibial anterior. Lima et al. ${ }^{37}$ não observaram diferença significativa na quantidade de colágeno em animais com lesão no TCC e tratados com 3 e 6 J. O estudo de Pallotta et al. ${ }^{25}$ com diferentes doses de energia (1, 3, 6 e $10 \mathrm{~J}$ ) revelou que, para cada dose utilizada em um determinado tempo da lesão experimental (artrite na articulação fêmoropatelar), existe uma resposta satisfatória. Os grupos que utilizaram doses de 6 e $10 \mathrm{~J}$ apresentaram redução do número de leucócitos e inibição da atividade das MMPs articulares. Também ocorreu redução do infiltrado de neutrófilos nos grupos que utilizaram laserterapia nas doses de 3, 6 e $10 \mathrm{~J}$, e aumento das COX-1 e 2 nos tratados com 3 e 10 J. Portanto, essa variação de utilização de comprimento de onda e dose de energia é um aspecto complicador demonstrado nesta revisão sistemática.

O comprimento de onda é uma variável que deve ser utilizada de acordo com o tecido biológico que receberá a radiação, pois está diretamente relacionado com a característica do tecido e a profundidade em que se encontra. Barbosa et al. ${ }^{38}$ não observaram diferença nos resultados obtidos comparando grupos tratados com diferentes comprimentos de onda (660 ou $830 \mathrm{~nm}$ ). Da mesma forma, Moreira et al. ${ }^{42}$ também não demonstraram diferenças no comprimento de onda utilizado (830 ou 904 nm) para tratar lesão do músculo gastrocnêmio. De acordo com os autores, a deposição do colágeno foi semelhante em ambos os grupos. Da mesma forma que mencionado para a dose de energia utilizada, o comprimento de onda é dose-dependente para o tratamento de tendinopatia do TCC ${ }^{32}$. Diferentes comprimentos de onda agem por mecanismos distintos, e influenciam a absorção e difusão da radiação ${ }^{7}$. Comprimentos de onda na faixa de 600 a $700 \mathrm{~nm}$ são indicados para o tratamento de tecidos localizados mais superficialmente, enquanto os entre 780 a $950 \mathrm{~nm}$ são mais indicados nas lesões que atingem estruturas mais profundas, devido ao maior poder de penetração. Por outro lado, comprimentos entre 700 e 770 nm não possuem uma atividade adequada. Na presente revisão sistemática, não foram encontrados experimentos que utilizaram comprimentos de onda entre 700 e $770 \mathrm{~nm}$ que se enquadrassem dentro dos critérios de inclusão adotados. Entretanto, nove (30\%) estudos utilizaram comprimentos de onda entre 632,8 e 670 nm em TCC $22-24,31,37,38$ e tibial anterior ${ }^{12,45}$.

Em estudo realizado por Silva et al. ${ }^{56} \mathrm{em}$ TCC de ratos Wistar, utilizando 3 J de energia e 904 nm de comprimento de onda, a laserterapia apresentou melhor resposta (menor reação inflamatória e aderência fibrinosa, e maior quantidade de fibras colágenas) se associada à caminhada em esteira, que é um exercício excêntrico. Nesse tipo de exercício, o músculo contraído é alongado enquanto produz força gerada durante a contração. Essa força não é suficiente para vencer a carga imposta, ocorrendo o alongamento das fibras ${ }^{57}$, o que resulta em importantes alterações na capacidade e massa muscular.

O uso de apenas quatro bases de busca pode ter sido um fator limitante no presente artigo. É possível, que estudos controlados e de qualidade não tenham sido incluídos. Por outro lado, para a escolha das bases foi levado em consideração outros estudos sistemáticos, e os critérios para publicação de artigos científicos. Ainda com relação aos aspectos relacionados com as limitações dessa revisão sistemática, é necessário comentar de alguns vieses clássicos que podem ocorrer nesse tipo de investigação, com destaque para os de seleção, duplicação e o de condução. Medidas previamente mencionadas, utilizando adequada metodologia, foram utilizadas para evitar esses vieses. Entre elas pode-se destacar a própria base de dados incluídas (Medline, Lilacs, PEDro e SciELO), consideradas altamente confiáveis. A seleção dos estudos se baseou inicialmente no título e resumo/ abstract, que foi seguido pelo material e métodos da pesquisa. Adicionalmente, a realização do registro dos artigos no período de 2005 a 2013 por dois pesquisadores independentes, com posterior retirada dos em duplicata, foi uma forma de reduzir mais um possível vies. Finalmente, é importante destacar que um dos principais vies relacionados com uma revisão sistemática sobre um determinado tema é a condução dos experimentos. Embora tenham sido incluídos apenas estudos experimentais randomizados e controlados, foi encontrada uma grande variedade na metodologia empregada. Mesmo havendo uma recomendação da Associação Mundial para Terapia com Laser sobre a dose de energia adequada para cada comprimento de onda, respeitando um determinado período de tempo e a afecção a ser tratada, foi possível perceber uma importante variabilidade nas doses utilizadas nos estudos apresentados nessa revisão sistemática. Isso ocorre porque ainda existem dúvidas sobre a dose e o comprimento de onda ideal, que devem ser esclarecidas com mais estudos sobre o tema em diferentes tecidos e fases do processo de reparação. Nesse contexto, o leitor deverá ser capaz de perceber que metodologias diferentes podem gerar resultados diferentes e, portanto, contraditórios. Entretanto, de uma forma geral, embora os estudos demonstrem incertezas, pode-se destacar que existe boa evidência para suportar a recomendação da laserterapia com a finalidade de redução da reação inflamatória excessiva que ocorre na fase inicial de uma lesão ortopédica aguda.

\section{CONSIDERAÇÕES FINAIS}

Independentemente da variabilidade encontrada entre os estudos científicos incluídos nessa revisão sistemática, a maioria das pesquisas sinalizam efeitos positivos da terapia, principalmente na modulação da resposta inflamatória em lesões agudas, associadas ou não a outros tratamentos. Por outro lado, o ideal é que pesquisas de qualidade, realizadas de forma randomizada, continuem sendo conduzidas para que a dose e o comprimento de onda sejam mais bem definidos para cada afecção ortopédica. Nesse sentido, seria importante que outras modalidades fisioterapêuticas pudessem ser associadas à laserterapia, como, por exemplo, o exercício excêntrico controlado, que sabidamente é fundamental para o retorno à funcionalidade dos diversos problemas relacionados com o aparelho locomotor.

\section{AGRADECIMENTOS}

Os autores agradecem a Coordenação de Aperfeiçoamento de Pessoal de Nível Superior (CAPES) por disponibilizar bolsa de estudo, a Fundação de Amparo à Pesquisa do estado de Minas Gerais (FAPE$\mathrm{MIG}$ ) pelo suporte financeiro e, principalmente, ao Conselho Nacional de Desenvolvimento Científico e Tecnológico (CNPq) por financiar o projeto de pesquisa (no. processo 472832/2012-3) para qual foi originada essa revisão sistemática.

Todos os autores declararam não haver qualquer potencial conflito de interesses referente a este artigo.

CONTRIBUIÇÕES DOS AUTORES: Cada autor contribuiu individual e significantemente para o desenvolvimento deste artigo. MOS (0000-0002-2951-1950)* realizou a revisão de literatura. MVS $(0000-0002-2656-3333)^{*}$ e MOS realizaram a redação do manuscrito e avaliação dos dados. MVS realizou a revisão de toda a parte escrita e fez todas as adequações necessárias, contribuindo assim com o conceito intelectual do estudo e foi a responsável não somente para o envio do manuscrito, mas também pelas correções sugeridas pelos revisores. *Número ORCID (Open Researcher and Contributor ID). 


\section{REFERÊNCIAS}

1. Andres BM, Murrell GA. Treatment of tendinopathy: what works, what does not, and what is on the horizon. Clin Orthop Relat Res. 2008;466(7):1539-54.

2. Baroni BM, Leal Junior EC, De Marchi T, Lopes AL, Salvador M, Vaz MA. Low level laser therapy before eccentric exercise reduces muscle damage markers in humans. Eur J Appl Physiol. 2010;110(4):789-96.

3. Wood VT, Pinfildi CE, Neves MA, Parizoto NA, Hochman B, Ferreira LM. Collagen changes and realignment induced by low-level laser therapy and low-intensity ultrasound in the calcaneal tendon. Lasers Surg Med. 2010;42(6):559-65.

4. Xavier M, David DR, de Souza RA, Arrieiro AN, Miranda H, Santana ET, et al. Anti-inflammatory effects of low-level light emitting diode therapy on Achilles tendinitis in rats. Lasers Surg Med. 2010;42(6):553-8.

5. Abate M, Silbernagel KG, Siljeholm C, Di lorio A, De Amicis D, Salini V, et al. Pathogenesis of tendinopathies: inflammation or degeneration? Arthritis Res Ther. 2009;11(3):235.

6. Fillipin LI, Mauriz JL, Vedovelli K, Moreira AJ, Zettler CG, Lech O, et al. Low-level laser therapy (LLLT) prevents oxidative stress and reduces fibrosis in rat traumatized Achilles tendon. Lasers Surg Med. 2005;37(4):293-300

7. Hamblin MR, Demidova TN. Mechanisms of low level light therapy. Proc SPIE. 2006;6140:1-12.

8. Matić M, Lazetić B, Poljacki M, Duran V, Ivkov-Simić M. Low level laser irradiation and its effect on repair processes in the skin. Med Pregl. 2003;56(3-4):137-41.

9. Pires D, Xavier M, Araújo T, Silva JA Jr, Aimbire F, Albertini R. Low-level laser therapy (LLLT; 780 nm) acts differently on mRNA expression of anti- and pro-inflammatory mediators in an experimental model of collagenase-induced tendinitis in rat. Lasers Med Sci. 2011;26(1):85-94.

10. Vladimirov YA, Osipov AN, Klebanov GI. Photobiological principles of therapeutic applications of laser radiation. Biochemistry (Mosc). 2004;69(1):81-90.

11. Gur A, Sarac AJ, Cevik R, Altindag O, Sarac S. Efficacy of $904 \mathrm{~nm}$ gallium arsenide low level laser therapy in the management of chronic myofascial pain in the neck: a double-blind and randomize-controlled trial. Lasers Surg Med. 2004;35(3):229-35

12. Lopes-Martins RA, Marcos RL, Leonardo PS, Prianti AC Jr, Muscará MN, Aimbire F, et al. Effect of lowlevel laser (Ga-Al-As $655 \mathrm{~nm}$ ) on skeletal muscle fatigue induced by electrical stimulation in rats. J Appl Physiol (1985). 2006;101(1):283-8.

13. Pretel H, Lizarelli RF, Ramalho LT. Effect of low-level laser therapy on bone repair: histological study in rats. Lasers Surg Med. 2007;39(10):788-96.

14. Assis L, Moretti Al, Abrahão TB, de Souza HP, Hamblin MR, Parizotto NA. Low-level laser therapy ( $808 \mathrm{~nm}$ ) contributes to muscle regeneration and prevents fibrosis in rat tibialis anterior muscle after cryolesion. Lasers Med Sci. 2013;28(3):947-55.

15. Hawkins D, Abrahamse H. Phototherapy - a treatment modality for wound healing and pain relief. Review. African J Biomed Res. 2007;10(2):99-9.

16. Ihsan FR. Low-level laser therapy accelerates collateral circulation and enhances microcirculation. Photomed Laser Surg. 2005;23(3):289-94.17.

17. Lins RDAU, Dantas EM, Lucena KCR, Catão MHCV, Granville-Garcia AF, Carvalho Neto LG. Efeitos bioestimulantes do laser de baixa potência no processo de reparo. An Bras Dermatol. 2010;85(6):849-55.

18. Medrado AR, Pugliese LS, Reis SR, Andrade ZA. Influence of low level laser therapy on wound healing and its biological action upon myofibroblasts. Lasers Surg Med. 2003;32(3):239-44.

19. Pereira AN, Eduardo Cde P, Matson E, Marques MM. Effect of low-power laser irradiation on cell growth and procollagen synthesis of cultured fibroblasts. Lasers Surg Med. 2002;31(4):263-7.

20. Woodruff LD, Bounkeo JM, Brannon WM, Dawes KS, Barham CD, Waddell DL, et al. The efficacy of laser therapy in wound repair: a meta-analysis of the literature. Photomed Laser Surg. 2004;22(3):241-7.

21. Bjordal JM, Lopes-Martins RA, Joensen J, Couppe C, Ljunggren AE, Stergioulas A, et al. A systematic review with procedural assessments and meta-analysis of low level laser therapy in lateral elbow tendinopathy (tennis elbow). BMC Musculoskelet Disord. 2008;9:75.

22. Arruda ERB, Rodrigues NC, Taciro C, Parizotto NA. Influência de diferentes comprimentos de onda da laserterapia de baixa intensidade na regeneração tendínea de rato após tenotomia. Rev Bras Fisioter. 2007;11(4):283-8

23. Laraia EM, Silva IS, Pereira DM, dos Reis FA, Albertini R, de Almeida P, et al. Effect of low-level laser therapy $(660 \mathrm{~nm})$ on acute inflammation induced by tenotomy of Achilles tendon in rats. Photochem Photobiol. 2012;88(6):1546-50

24. Nouruzian M, Alidoust M, Bayat M, Bayat M, Akbari M. Effect of low-level laser therapy on healing of tenotomized Achilles tendon in streptozotocin-induced diabetic rats. Lasers Med Sci. 2013;28(2):399-405

25. Pallotta RC, Bjordal JM, Frigo L, Leal Junior EC, Teixeira S, Marcos RL, et al. Infrared (810-nm) low-level laser therapy on rat experimental knee inflammation. Lasers Med Sci. 2012;27(1):71-8.

26. Ramos L, Leal Junior EC, Pallotta RC, Frigo L, Marcos RL, et al. Infrared ( $810 \mathrm{~nm}$ ) low-level laser therapy in experimental model of strain-induced skeletal muscle injury in rats: effects on functional outcomes. Photochem Photobiol. 2012;88(1):154-60,

27. Lake SP, Ansorge HL, Soslowsky LJ. Animal models of tendinopathy. Disabil Rehabil. 2008;30(2022): $1530-41$

28. Warden SJ. Animal models for the study of tendinopathy. Br J Sports Med. 2007;41(4):232-40.

29. Lui PP, Maffulli N, Rolf C, Smith RK. What are the validated animal models for tendinopathy? Scand J Med Sci Sports. 2011;21(1):3-17.

30. Moher D, Liberati A, Tetzlaff J, Altman DG. The PRISMA statement. PloS Med. 2009; 6(6):E10000097. Disponível em: http://www.prisma-statement.org/2.1.4\%20-\%20PRISMA\%20Flow\%202009\%20 Diagram.pdf. [Acessado em 18 outubro, 2015].
31. Salate AC, Barbosa G, Gaspar P, Koeke PU, Parizotto NA, Benze BG, et al. Effect of In-Ga-Al-P diode laser irradiation on angiogenesis in partial ruptures of Achilles tendon in rats. Photomed Laser Surg. 2005;23(5):470-5

32. Oliveira FS, Pinfildi CE, Parizoto NA, Liebano RE, Bossini PS, Garcia EB, et al. Effect of low level laser therapy $(830 \mathrm{~nm})$ with different therapy regimes on the process of tissue repair in partial lesion calcaneous tendon. Lasers Surg Med. 2009;41(4):271-6.

33. Marcos RL, Leal-Junior EC, Arnold G, Magnenet V, Rahouadj R, Wang X, et al. Low-level laser therapy in collagenase-induced Achilles tendinitis in rats: analyses of biochemical and biomechanical aspects. J Orthop Res. 2012;30(12):1945-51.

34. Casalechi HL, Leal-Junior EC, Xavier M, Silva JA Jr, de Carvalho Pde T, Aimbire F, et al. Low-level laser therapy in experimental model of collagenase-induced tendinitis in rats: effects in acute and chronic inflammatory phases. Lasers Med Sci. 2013;28(3):989-95.

35. Guerra Fda R, Vieira CP, Almeida MS, Oliveira LP, de Aro AA, Pimentel ER. LLLT improves tendon healing through increase of MMP activity and collagen synthesis. Lasers Med Sci. 2013;28(5):1281-8.

36. Joensen J, Gjerdet NR, Hummelsund S, Iversen V, Lopes-Martins RA, Bjordal JM. An experimental study of low-level laser therapy in rat Achilles tendon injury. Lasers Med Sci. 2012;27(1):103-11.

37. Lima JGM, Filho Oliveira GR, Lima MTBR, Ferreira AS, Silva JG. Influence of low intensity laser therapy (AsGa) on the cicatrization process of mechanic tendon injury in Wistar rats. Laser Physics. 2012;22(9):1445-8.

38. Barbosa D, de Souza RA, de Carvalho WR, Xavier M, de Carvalho PK, Cunha TC, et al. Low-level laser therapy combined with platelet-rich plasma on the healing calcaneal tendon: a histological study in a rat model. Lasers Med Sci. 2013;28(6):1489-94.

39. Rizzi CF, Mauriz JL, Freitas Corrêa DS, Moreira AJ, Zettler CG, Filippin LI, et al. Effects of low-level lase therapy (LLLT) on the nuclear factor (NF)-kappaB signaling pathway in traumatized muscle. Lasers Surg Med. 2006;38(7):704-13.

40. Leal Junior EC, Lopes-Martins RA, de Almeida P, Ramos L, Iversen WV, Bjordal JM. Effect of low-leve laser therapy (GaAs $904 \mathrm{~nm}$ ) in skeletal muscle fatigue and biochemical markers of muscle damage in rats. Eur J Appl Physiol. 2010;108(6):1083-8.

41. Falcai MJ, Monte-Raso W, Okubo R, Zamarioli A, Carvalho LC, Shiamano AC. Análise biomecânica e histológica de músculos gastrocnêmio de ratas submetidas à lesão muscular e tratadas com laserterapia de baixa intensidade. Rev Bras Ortop. 2010;45(4):444-8.

42. Moreira FF, Oliveira ELP, Barbosa FS, Silva JG. Laserterapia de baixa intensidade na expressão de colágeno após lesão muscular cirúrgica. Fisioter Pesqui. 2011;18(1):37-2.

43. Silveira PC, da Silva LA, Pinho CA, De Souza PS, Ronsani MM, Scheffer Dda L, et al. Effects of low-leve laser therapy (GaAs) in an animal model of muscular damage induced by trauma. Lasers Med Sci. 2013;28(2):431-6.

44. de Paiva Carvalho RL, Leal-Junior EC, Petrellis MC, Marcos RL, de Carvalho MH, De Nucci G, et al. Effects of low-level laser therapy (LLLT) and diclofenac (topical and intramuscular) as single and combined therapy in experimental model of controlled muscle strain in rats. Photochem Photobiol. 2013;89(2):508-12.

45. Rodrigues NC, Brunelli R, de Araújo HS, Parizotto NA, Renno AC. Low-level laser therapy (LLLT) (660nm) alters gene expression during muscle healing in rats. J Photochem Photobiol B. 2013;120:29-35.

46. Fangel R, Bossini PS, Renno AC, Ribeiro DA, Wang CC, Toma RL, et al. Low-level laser therapy, at 60 $\mathrm{J} / \mathrm{cm} 2$ associated with a Biosilicate $\left.{ }^{(}\right)$increase in bone deposition and indentation biomechanica properties of callus in osteopenic rats. J Biomed Opt. 2011;16(7):078001.

47. Alves AC, de Carvalho PT, Parente M, Xavier M, Frigo L, Aimbire F, et al. Low-level laser therapy in different stages of rheumatoid arthritis: a histological study. Lasers Med Sci. 2013;28(2):529-36.

48. Fernandes KR, Ribeiro DA, Rodrigues NC, Tim C, Santos AA, Parizotto NA, et al. Effects of low-level laser therapy on the expression of osteogenic genes related in the initial stages of bone defects in rats. Biomed Opt. 2013;18(3):038002.

49. Schultz GS, Laswig G, Wysocki A. Extracellular matrix: review of its roles in acute and chronic wounds World Wide Wounds. 2005. Disponível em: http://www.worldwidewounds.com/2005/august/ Schultz/Extrace-Matric-Acute-Chronic-Wounds.html. [Acessado em 3 Julho, 2013].

50. Marcu KB, Otero M, Olivotto E, Borzi RM, Goldring MB. NF-kappaB signaling: multiple angles to target OA. Curr Drug Targets. 2010;11(5):599-613.

51. Frangogiannis NG, Youker KA, Rossen RD, Gwechenberger M, Lindsey MH, Mendoza LH, et al. Cytokines and the microcirculation in ischemia and reperfusion. J Mol Cell Cardiol. 1998;30(12):2567-76.

52. Pavesi VCS. Efeito da criolesão no remodelamento da matriz extracellular em músculo esquelético de ratos [dissertação]. São Paulo: Universidade Nove de Julho. Programa de Pós-Graduação em Ciências da Reabilitação; 2009

53. Zamdim BM, Souza MV, Frassy LN, Vilória MIV, Maia L, Fonseca CC, et al. Immunohistochemistry of factor VIII, histology and morphometry in equine tendon treated with platelet-rich plasma. Rev Bras Med Vet. 2013;35(2):169-84.

54. Souza MV, Pinto J O, Costa MBM, Alves MS, Silva MO, Martinho KO, et al. Expressão gênica do colágeno em ferida cutânea de equinos tratada com plasma rico em plaquetas. Pesq Vet Bras. 2014;34(3):233-40

55. Souza MV, Silva MB, Pinto JO, Lima MBS, Crepaldi J, Lopes GFM, et al. Immunohistochemical expression of collagens in the skin of horses treated with leukocyte-poor platelet-rich Plasma. Biomed Res Int. 2015;2015:893485

56. Silva MO, Costa MBM, Borges APB, Dornas RF, Moreira JCL, Souza MV. Indução de tendinopatia em ratos Wistar: modelo experimental. Rev Acad Ciênc Agrár Ambient. 2013;11(3):1-12.

57. Frick A. Stretching exercise for horses: are they effective? J Equine Vet Sci. 2010;30(1):50-9. 